\title{
The value of molecular markers in classification and prediction of progression in non-muscle-invasive bladder cancer
}

\author{
Chin-Chen Pan \\ Department of Pathology and Laboratory Medicine, Taipei Veterans General Hospital, Taipei, Taiwan \\ Correspondence to: Chin-Chen Pan, MD. Department of Pathology and Laboratory Medicine, Taipei Veterans General Hospital, Taipei 11217, \\ Taiwan. Email: ccpan@vghtpe.gov.tw. \\ Provenance: This is a Guest Editorial commissioned by Section Editor Xiao Li (Department of Urology, Jiangsu Cancer Hospital \& Jiangsu Institute \\ of Cancer Research \& Nanjing Medical University Affiliated Cancer Hospital, Nanjing, China). \\ Comment on: van Kessel KEM, van der Keur KA, Dyrskjøt L, et al. Molecular Markers Increase Precision of the European Association of Urology \\ Non-Muscle-Invasive Bladder Cancer Progression Risk Groups. Clin Cancer Res 2018;24:1586-93.
}

Submitted Jul 16, 2018. Accepted for publication Jul 23, 2018.

doi: $10.21037 /$ tau.2018.07.11

View this article at: http://dx.doi.org/10.21037/tau.2018.07.11

Urothelial carcinoma is the most common type of bladder cancer. The majority of urothelial carcinomas are lowgrade, papillary, non-muscle invasive bladder cancer (NMIBC) treated by transurethral resection followed by intravesical instillation (1). The remaining carcinomas consist of high-grade, muscle-invasive tumors that are eligible for cystectomy. A significant proportion of NMIBC recur following TUR, and a minority will progress into high-grade tumors that are either muscle-invasive or metastatic tumor (2). For NMIBC treated by TUR, diligent follow-up plans are paramount to detect recurrence and progression.

However, the accurate designation of NMIBC that will progress to muscle invasion has yet to be perfected and currently relies on surveillance of regular cystoscopy. Cystoscopy is an invasive procedure causing discomfort to the patients. The long survival of NMIBC patients makes bladder cancer one of the costliest cancers (3). Costs and patient burden could be reduced by lowering the number of cystoscopies in patients with very low risk NMIBC (4). At the other end of the prognostic spectrum, additional prognostic indicators that are able to predict a subset of NMIBCs likely to progress are needed to tailor more aggressive surveillance and management. Therefore, risk stratification is imperative for classifying patients with similar risks of recurrence and progression, and it helps to determine the appropriate management protocol for each risk category, and also a personalized follow-up and therapeutic strategy for individual patient. Given the disparate prognoses and treatment plans, a prognostic model involving multiple factors present at initial diagnosis will be helpful.

The European Association of Urology (EAU) has developed guidelines (5) for the treatment and followup of patients with NMIBC to stratify them into low-, intermediate-, and high-risk groups. These guidelines are based on a risk scoring table derived from 2,596 patients from European Organization for Research and Treatment (EORTC) of Cancer trials (2). With six clinicopathological factors (number of tumors, tumor size, prior recurrence rate, T stage, carcinoma in situ (CIS) and 1973 WHO grade), a concordance index of 0.66 for predicting recurrence and 0.75 for predicting progression at 5 years could be achieved.

The main limitation of the EORTC risk tables is that the patients were recruited between 1979 and 1989; hence they were mostly treated with intravesical chemotherapy regimens differing from current practice. For example, the use of a single, immediate chemotherapeutic instillation, induction and maintenance BCG, and repeat transurethral resection of the bladder tumor were not considered in the development of these risk tables (1). Improvements in chemotherapy administration, in addition to the increased use of BCG, may reduce the predictability of these tables. In addition, very few cases of CIS were included in the studies that would weaken its predictive power. 
The EORTC table requires clinical information not necessarily available for every patient, such as tumor multiplicity and size, the information of which could only be acquired through detailed documentation by surgeons. Whether the surgeon performs biopsy on non-tumorous part of mucosa will affect the determination of the presence of concomitant CIS. The EORTC table adopts old 1973 WHO grading system, while the grading system has been revised since 2004 WHO classification (6). Unfortunately, there are no one-by-one translations between the new and old grades (1). Although the EORTC table has been widely used in Europe, it has not gained full acceptance in the United States and elsewhere as yet.

With better understanding about tumor biology and assistance of state-of-the-art technologies, molecular markers have been extensively evaluated as adjuncts for the purposes of classification and prognostication. Identification of highly sensitive and specific biomarkers for prediction of outcome for patients with NMIBC is of utmost clinical importance. Tumors with similar histopathological characteristics can have widely different molecular features and may belong to distinct molecular subgroups with different clinical risks. Consequently, clinically useful molecular tests for stratifying patients to treatment and follow-up regimens beyond wellestablished clinical risk factors are greatly needed. These molecular markers are expected to reflect genetic and phenotypic hallmarks more directly than histomorphology, and the results of the tests can be interpreted by more objective criteria than histopathology which is often subject to inter-observer variation.

It has been well established that bladder tumors arise through at least two divergent mechanisms (7). Somatic mutations in the FGFR 3 accompanied by losses of chromosome 9 are more frequent in low-grade NMIBC, while TP53 mutations are associated with highgrade, muscle-invasive bladder cancer $(8,9)$. Other gene mutations or dysregulation including tumor suppressor genes, oncogenes, cell cycle regulators, growth factors and receptors, cell adhesion molecules, and homeostatic modulators may confer additional growth advantage to the tumors $(10,11)$. Some of the markers may have prognostic impact as demonstrated in small series; however, they are rarely discriminatory enough for clinical use as individual markers. On the other hand, combining the makers with clinical data into a combinatory score may enhance the prognostic power. An immunohistochemical panel consisting of Ki-67, p53, cyclin D1, COX2 and HSP27 can segregate a subset with high aberrant index from non-invasive and focally invasive NMIBC that behaves as aggressive as those with extensive invasion. Thus, the biomarker testing can discern an aggressive subset of NMIBC even before the tumors manifest recognizable extensive lamina propria invasion (12).

Microarray and integrated next generation sequencing provide efficient platforms for simultaneous examination of a huge number of genes. Dyrskjøt et al. used cDNA microarray to delineate a 45 -gene signature for high likelihood of muscle-invasive progression in NMIBC (13). Later in a multicenter study they developed 52-gene, 88gene, and 68-gene classifiers to correlate with pathologic stage, progression and CIS, respectively (14). Fristrup et al. further chose four crucial markers (cyclin D1, MCM7, TRIM29, UBE2C) from the dataset to predict progression by using immunohistochemistry. The four-marker panel could separate patients with NMIBC into 3-tier risk groups (15). These signatures were transferred into a 12gene real-time quantitative polymerase chain reaction (PCR) assay that differentiated high versus low risk, and was validated in a multicenter prospective study (16).

Alternately, several studies have attempted molecular classification. Lindgren et al. employed hierarchical cluster analyses on cDNA array to identify two intrinsic molecular subtypes that showed significant correlation with disease-free survival (17). Using global gene expression analysis, Sjödahl et al. discerned 28 markers to classify urothelial carcinoma into urothelial-like, genomically unstable, basal/SCC-like, mesenchymal-like, and smallcell/neuroendocrine-like subtypes $(18,19)$, that roughly corresponded to low-grade papillary, high-grade, squamous, sarcomatoid and neuroendocrine differentiation. Choi et al. defined a distinct luminal type enriched with activating FGFR3 mutations, and a basal type which is characterized by squamous differentiation and basal subtypes (20). The basal type was associated with shorter survival than the luminal type. The Cancer Genome Atlas Research Network (21) meanwhile disclosed similar findings (called papillarylike and basal/squamous-like). Although these analyses investigated bladder cancer as a whole, the observations can be applied to NMIBC also. Even the tumor initially presents in $\mathrm{Ta} / \mathrm{T} 1$, an unfavorable outcome is foreseen if the tumor expresses an aggressive molecular signature.

The article of van Kessel et al. (22) discussed in this editorial is a timely one. The strength of the cohort is its large sample size, its prospective nature, and its multiinstitutional and multinational character (1,239 patients in 6 European countries). The markers the authors analyzed 
included GATA2, TBX2, TBX3, and ZIC4 for methylation status and FGFR3, TERT, PIK3CA, and RAS for mutation status. The methylation markers were chosen according to a previous genome-wide analysis of $\mathrm{CpG}$ island methylation in bladder cancer (23). The findings were integrated into the EORTC table. The combination of EAU risk category, FGFR 3 mutation status, GATA2, and TBX3 methylation status resulted in an overall concordance of 0.76 , that was higher than the 0.72 of biomarker alone, and the 0.70 of EAU risk category alone. The authors proposed to refine the EAU risk category. By the use of GATA2 and $F G F R 3$, the EAU high-risk group could be reclassified into a good class (GATA2 unmethylated, FGFR3 mutated), a poor class (GATA2 methylated, FGFR3 wild type) and an otherwise moderate class; thus these patients could be assigned to different management strategies accordingly. It demonstrates that molecular testing is not just of academic interest, but truly has contribution in clinical management.

Some issues of the study should be addressed. The authors aimed at improving the EAU guideline and have succeeded to attain the goal by adding those molecular markers. However, the limitations inherited in the preexisting EORTC table were not resolved. The study mainly focused on the EAU high-risk group. The alterations of the makers in the cases which progressed into muscle invasion in the EAU low- and intermediate-risk groups were not revealed. Is it possible that those progressors actually represented the poor class imprecisely allocated to the low/ intermediate-risk categories? Therefore, these markers might still have merit to test for the low/intermediate-risk groups. Moreover, the markers might not necessarily be the optimal ones. Novel biomarkers which are more potent may replace the current ones in the future.

Finally, the benefit of a prognostic model must be weighed against the cost and labor of the tests. For practical consideration, the number of variables in the model had better be kept as few as possible yet without sacrificing the robustness. Microarray gene expression profiling and experiments on dozens of genes are difficult to translate into clinical tools given the complexity of the assays that require time and expertise. Such demanding tests are beyond the capacity of ordinary clinical and pathologic laboratories so they can only be undertaken in central laboratories or commercial companies that unavoidably entail much inconvenience and expenditure. The study of van Kessel et al. is impressive for the required markers were cut down to merely two (GATA2 and FGFR3). The authors claimed that those assays are inexpensive; yet I still have concern over the feasibility of the assays (bisulfate-specific PCR, SNaPshot, etc.) in usual laboratories. Given the large case burden of NMIBC, I doubt that many laboratory directors are willing to implement the tests for routine use even restricting to the high-risk group. Despite these limitations, van Kessel and colleagues have offered a sound approach for constructing a prognostic model that incorporates molecular markers, and opened an avenue especially toward precision medicine.

\section{Acknowledgements}

None.

\section{Footnote}

Conflicts of Interest: The author has no conflicts of interest of declare.

\section{References}

1. Colombel M, soloway M, Akaza H, et al. Epidermiology, staging, grading, and risk stratification of bladder cancer. Eur Urol 2008;7:618-26.

2. Sylvester RJ, van der Meijden AP, Oosterlinck W, et al. Predicting recurrence and progression in individual patients with stage Ta $\mathrm{T} 1$ bladder cancer using EORTC risk tables: a combined analysis of 2596 patients from seven EORTC trials. Eur Urol 2006;49:466-5; discussion 475-7.

3. Botteman MF, Pashos CL, Hauser RS, et al. Quality of life aspects of bladder cancer: a review of the literature. Qual Life Res 2003;12:675-88.

4. van der Aa MN, Steyerberg EW, Sen EF, et al. Patients' perceived burden of cystoscopic and urinary surveillance of bladder cancer: a randomized comparison. BJU Int 2008;101:1106-10.

5. Babjuk M, Böhle A, Burger M, et al. EAU Guidelines on Non-Muscle-invasive Urothelial Carcinoma of the Bladder: Update 2016. Eur Urol 2017;71:447-61.

6. Eble JN. World Health Organization Classification of Tumours. Pathology and Genetics of Tumours of the Urinary System and Male Genital Organs. 2004. Available online: https://www.iarc.fr/en/publications/pdfs-online/ pat-gen/bb7/BB7.pdf

7. Wu XR. Urothelial tumorigenesis: a tale of divergent pathways. Nat Rev Cancer 2005;5:713-25.

8. Hernández S, López-Knowles E, Lloreta J, et al. FGFR3 
and Tp53 mutations in T1G3 transitional bladder carcinomas: independent distribution and lack of association with prognosis. Clin Cancer Res 2005;11:5444-50.

9. van Rhijn BW, Lurkin I, Radvanyi F, et al. The fibroblast growth factor receptor 3 (FGFR3) mutation is a strong indicator of superficial bladder cancer with low recurrence rate. Cancer Res 2001;61:1265-8.

10. Cheng L, Zhang S, MacLennan GT, et al. Bladder cancer: translating molecular genetic insights into clinical practice. Hum Pathol 2011;42:455-81.

11. Proctor I, Stoeber K, Williams GH. Biomarkers in bladder cancer. Histopathology 2010;57:1-13.

12. Pan CC, Yu HJ, Chang YH. The prognostic value of combined clinicopathological and biomarker modelling for non-muscle-invasive bladder cancer. Histopathology 2014;65:207-15.

13. Dyrskjøt L, Zieger K, Kruhøffer M, et al. A molecular signature in superficial bladder carcinoma predicts clinical outcome. Clin Cancer Res 2005;11:4029-36.

14. Dyrskjøt L, Zieger K, Real FX, et al. Gene expression signatures predict outcome in non-muscle-invasive bladder carcinoma: a multicenter validation study. Clin Cancer Res 2007;13:3545-51.

15. Fristrup N, Birkenkamp-Demtröder K, Reinert T, et al. Multicenter validation of cyclin D1, MCM7, TRIM29, and UBE2C as prognostic protein markers in non-muscleinvasive bladder cancer. Am J Pathol 2013;182:339-49.

16. Dyrskjøt L, Reinert T, Algaba F, et al. Prognostic Impact of a 12-gene Progression Score in Non-muscle-invasive Bladder Cancer: A Prospective Multicentre Validation

Cite this article as: Pan CC. The value of molecular markers in classification and prediction of progression in non-muscleinvasive bladder cancer. Transl Androl Urol 2018;7(4):736-739. doi: $10.21037 /$ tau.2018.07.11
Study. Eur Urol 2017;72:461-9.

17. Lindgren D, Frigyesi A, Gudjonsson S, et al. Combined gene expression and genomic profiling define two intrinsic molecular subtypes of urothelial carcinoma and gene signatures for molecular grading and outcome. Cancer Res 2010;70:3463-72.

18. Sjödahl G, Lauss M, Lövgren K, et al. A molecular taxonomy for urothelial carcinoma. Clin Cancer Res 2012;18:3377-86.

19. Sjödahl G, Eriksson P, Liedberg F, et al. Molecular classification of urothelial carcinoma: global mRNA classification versus tumour-cell phenotype classification. J Pathol 2017;242:113-25.

20. Choi W, Porten S, Kim S, et al. Identification of distinct basal and luminal subtypes of muscle-invasive bladder cancer with different sensitivities to frontline chemotherapy. Cancer Cell 2014;25:152-65.

21. Cancer Genome Atlas Research Network. Comprehensive molecular characterization of urothelial bladder carcinoma. Nature 2014;507:315-22.

22. van Kessel KEM, van der Keur KA, Dyrskjøt L, et al. Molecular Markers Increase Precision of the European Association of Urology Non-Muscle-Invasive Bladder Cancer Progression Risk Groups. Clin Cancer Res 2018;24:1586-93.

23. Kandimalla R, van Tilborg AA, Kompier LC, et al. Genome-wide analysis of $\mathrm{CpG}$ island methylation in bladder cancer identified TBX2, TBX3, GATA2, and ZIC4 as pTa-specific prognostic markers. Eur Urol 2012;61:1245-56. 